\title{
Fibrin-collagen hydrogel as a scaffold for dermoepidermal skin substitute, preparation and characterization
}

\author{
Mahsa Mollapour Sisakht, a,b Mohammad Ali Nilforoushzadeh, a* Javad Verdi, a,c* Hamid Reza Banafshe, a,d* \\ Zahra Safaei Naraghi, ${ }^{, f}$ and Seyed Abdolreza Mortazavi-Tabatabaei ${ }^{9}$
}

\author{
applied Cell Sciences Department, Kashan University of Medical Science, Kashan, Iran. \\ bSkin and Stem Cell Research Center, Tehran University of Medical Sciences, Tehran, Iran. \\ 'Department of Tissue Engineering and Applied Cell Sciences, School of Advanced Technologies in Medicine, Tehran University of Medical Sciences, Tehran, Iran. \\ dPhysiology Research Center, Kashan University of Medical Sciences, Kashan, Iran. \\ eDepartment of Dermatology, Tehran University of Medical Sciences, Razi Hospital, Tehran, Iran. \\ fDepartment of Pathology, Tehran University of Medical Sciences, Razi Hospital, Tehran, Iran. \\ sProteomics Research Center, Shahid Beheshti University of Medical Sciences, Tehran, Iran. \\ *Correspondence to Mahsa Mollapour Sisakht (email: mahsamollapur@yahoo.com). \\ (Submitted: 02 October 2018 - Revised version received: 17 October 2018 - Accepted: 23 November 2018 - Published online: 26 February 2019)
}

\begin{abstract}
Objective Bioengineered skin substitutes were created to address wound healing problems. Skin substitutes contains live human cells seeded onto a matrix to provide cytokine, growth factor and other proteins from ECM required to decrease healing time. These products are classified based on their durability, the cells seeded on them, and their originality. In this study, we aimed to investigate fibrin-collagen hydrogel as a new scaffold to design a bilayer temporary skin equivalent.

Methods Fibrin gel was prepared by cross-linking fibrinogen with thrombin and mixing it with collagen type I. Human fibroblasts and keratinocytes were isolated from skin biopsies of healthy donors and foreskin and the cells were seeded onto three-dimensional hydrogel layer-by-layer. Morphological assessment, histological analysis, immunocytochemistry were performed to characterize the scaffold properties.

Results The results of scaffold characterization demonstrated good porosity, cell viability, and biocompatibility of the scaffold. Conclusion Fibrin-collagen as a natural material in organotypic cell culture modeling demonstrates that hydrogel scaffolds can be properly designed to generate bilayer or composite temporary skin grafts.

Keywords tissue engineering, fibrin-collagen, hydrogel, skin substitute
\end{abstract}

\section{Introduction}

Tissue engineering as a major component of regenerative medicine is now being marketed. Different types of product, especially in dermatology, are found globally. Despite its high cost, market studies show that the use of advanced wound dressing containing live skin cells has increased. ${ }^{1}$ Among the different types of skin substitutes, acellular dermal matrices have the biggest market share. ${ }^{2}$ Composite matrices derived from human keratinocytes, fibroblasts and bovine or porcine collagen developed by organogenesis are marketed as Apligraf. Orcel, TissueTech, and Theraskin composite products. ${ }^{3}$ Skin substitutes can be synthetic or natural. ${ }^{4}$ Of the synthetic and natural materials used to prepare skin substitute, hydrogels, and gels have advantages such as high exudate capacity, non-adherence, ease of removal from wounds, accelerated healing, pain and inflammation reduction, low cost, and easy development and handling ${ }^{5}$ for wound management.

Hydrogels are composites which are crosslinked by chemical or physical methods in water. They are characterized by remarkable water-absorbing swellability. ${ }^{6}$ Significant efforts have been made in research and development to commercialize hydrogels. Some hydrogel dressings synthesized by chemical cross-linking have appeared under the brand names of Geliperm ${ }^{\circledR}$, Curasol $^{\circledR}$, and Tegagel ${ }^{\circledR} .5$ The major drawback of hydrogels is their low mechanical stiffness, which can be improved by mixing or crosslinking with other polymers.

This study presents the preparation and characterization of a combination of collagen type I and fibrin as a natural composite polymer and assesses the ability of skin-cell seeding onto a scaffold to generate a permanent full-thickness or dermalepidermal skin equivalent. We hypothesize that the addition of type I collagen to fibrin will increase the mechanical features of fibrin. It is shown that this scaffold is suitable for three-dimensional (3D) cell cultures and it is possible for clinical application as a skin substitute.

\section{Materials and Methods Isolation and Culture of Fibroblast}

Human dermal fibroblasts were isolated from neonatal foreskin obtained at the time of circumcision, after receiving written informed consent from their parents. Samples were carried to the laboratory in DMEM medium containing $10 \%(\mathrm{v} / \mathrm{v})$ FBS and penicillin $(100 \mathrm{IU} / \mathrm{ml})$-streptomycin $(100 \mu \mathrm{g} / \mathrm{ml})$ (All of them from Gibco, Thermo Fisher Scientific). Before working with samples they were washed three times in washing solution, PBS (Gibco, Thermo Fisher Scientific) containing penicillin-streptomycin to remove of probably infection and any blood. Skin was cut into strips $1 \mathrm{~cm}$ wide using scalpel and this sample was incubated overnight in $4^{\circ} \mathrm{C}$ in dispase $0.1 \%$ (Gibco, Thermo Fisher Scientific) for easy separation of epidermal layer from dermal layer. The dermis was cut into very small pieces $\left(5 \mathrm{~mm}^{2}\right)$ using curved scissors and these pieces were transferred into a collagenase type II (200 $\mathrm{IU} / \mathrm{ml}$ ) (Gibco, Thermo Fisher Scientific) solution in an incubator at $37^{\circ} \mathrm{C}$ for $2 \mathrm{~h}$. Then, the suspension was centrifuged at $500 \mathrm{~g}$ for $5 \mathrm{~min}$. The pellet was resuspended in culture medium. 
The isolated cells were counted using hemocytometer (cell viability determined using trypan blue exclusion) and then seeded into culture dishes at cell density of $2 \times 105$ cells $/ \mathrm{cm}^{2}$ (Fig. 1A-1C).

The cells were cultured in a $\mathrm{CO}_{2}$ incubator at $37^{\circ} \mathrm{C}$ and the culture medium was changed twice a week. The cells were subcultured before they reached confluence by treating them with Trypsin-EDTA (Gibco, Thermo Fisher Scientific) for 5 min and a ratio of 1:3 was split into new dishes. ${ }^{8}$

\section{Isolation and Culture of Keratinocyte}

For separation of keratinocytes, the side up of skin was placed in $0.25 \%$ trypsin and then incubated at $37^{\circ} \mathrm{C}$ for $30 \mathrm{~min}$. Then, it was transferred into a $15-\mathrm{ml}$ centrifuge tube and was vortexed gently for $2 \mathrm{~min}$, and then the same volume of FBS was added to inhibit the action of the trypsin. The suspension was centrifuged at $500 \mathrm{~g}$ for $5 \mathrm{~min}$, the supernatant was discarded and the cell pellet was resuspended in Keratinocyte Growth
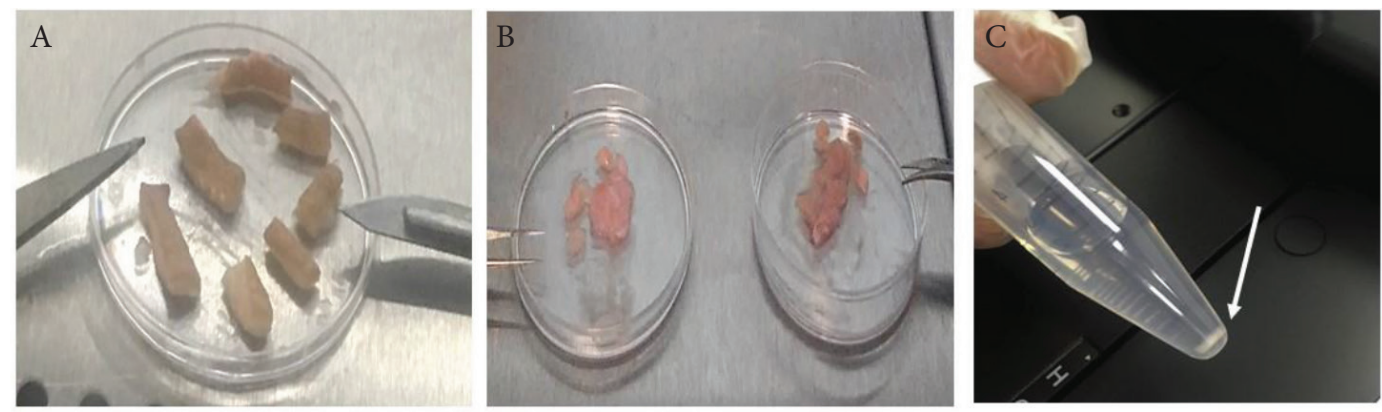

CK14

EpCAM-Dylight 488
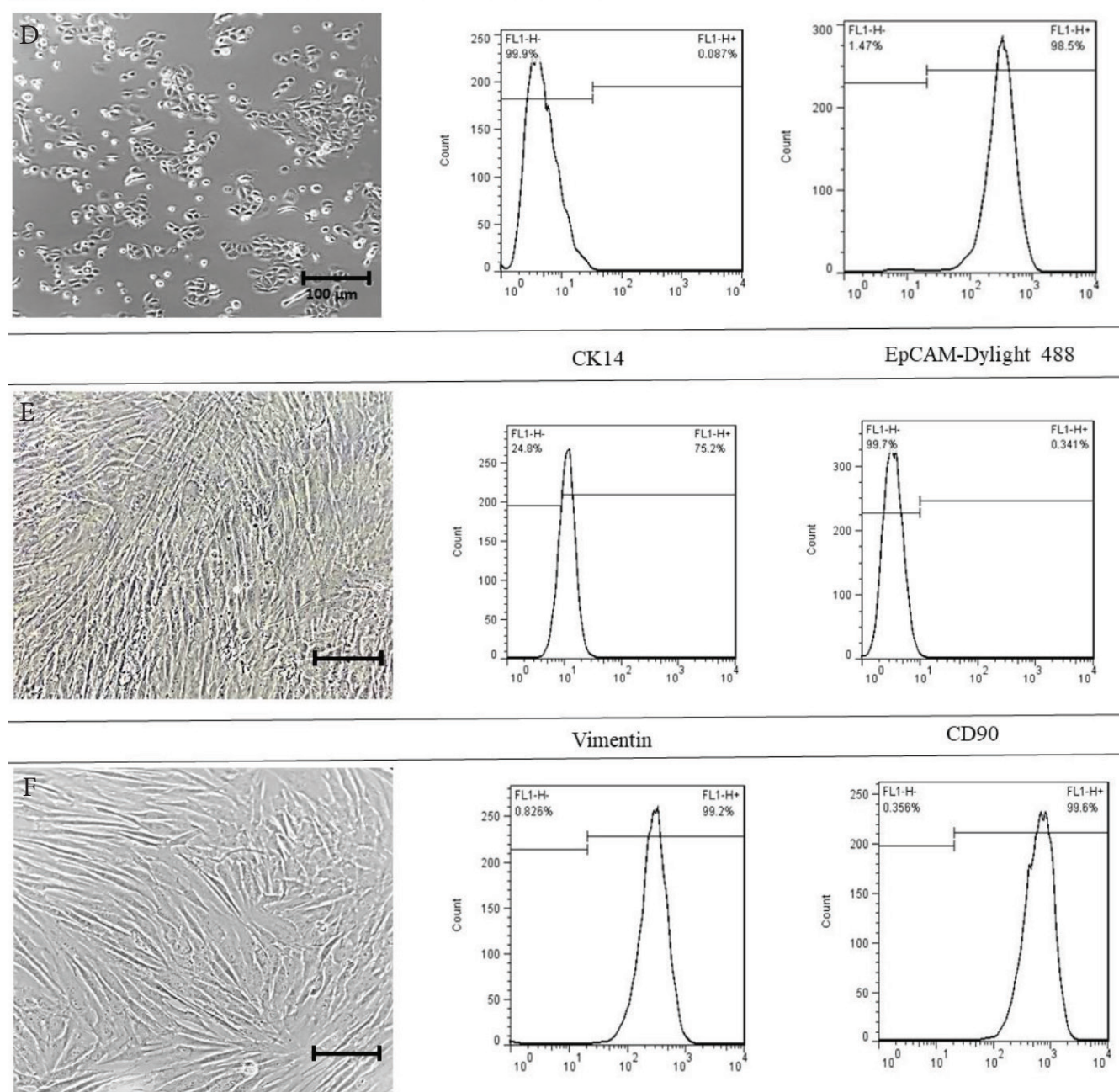

Fig. 1 Cells isolation and characterization. (A and B) Foreskin preparation for keratinocyte and fibroblast isolation. (C) White arrow shows cell pellet after centrifuge. (D) Expanded confluent Keratinocyte from cornified layer of epidermis cultured in KGM. All of these cells were stained for CK14 and EpCAM and analyzed by flow cytometry. Representative phase-contrast images of these cells seeded on tissue culture plastic (Right) (Scale bar: $100 \mu \mathrm{m}$ ). (E) Keratinocytes from basal layer of epidermis were stained for CD14 and EpCAM. (F) Fibroblasts were stained for Vimentin and CD90 and analyzed by flow cytometry. Phase-contrast micrograph of cells at passage three expanded on cell culture plastic (Scale bar: $100 \mu \mathrm{m})$. KGM, keratinocyte growth medium. 
Medium (Epilife Medium) (Gibco, Thermo Fisher Scientific). In addition, we used another cell culture media for keratinocyte cell culture. The cell pellet was resuspended in KGM-Gold Keratinocyte Growth Medium SingleQuot (Lonza, Sweden). Epidermal cells for both of them were attached within 24-48 h and the cells began to spread out on dish. The culture medium were changed daily and the dishes were confluent by $10-15$ days (Fig. 1A-1C).

\section{Characterization of Fibroblast and Keratinocyte}

For characterization of keratinocytes and fibroblast, common types of monoclonal antibodies were used consequently: antihuman EpCAM produced in mouse and cytokeratin 14 (CK14) for keratinocyte and CD90, Vimentin for fibroblast (all from BD Bioscience, USA). The cells were fixed using 4\% formaldehyde (Sigma-Aldrich, USA) and after three times of washing with PBS, antibodies were incubated on the cells for $1 \mathrm{~h}$ at $4^{\circ} \mathrm{C}$. The positive cells were detected using fluorescence microscope (Zeiss, Germany) (Fig. 1D-1F).

\section{Scaffold Preparation}

Fibrin hydrogel were made using human type I plasma fibrinogen at a concentration of $10 \mathrm{mg} / \mathrm{ml}$ in the presence of thrombin at a concentration of $100 \mathrm{U} / \mathrm{ml}$ (both from Sigma-Aldrich, USA) were used in a 3:1 ratio, respectively. The collagen gel mixture was prepared using rat tail type I gel (Sigma-Aldrich, USA), then collagen gel combined with $0.2 \mathrm{ml}$ neutralization buffer containing $0.15 \mathrm{M}$ sodium hydroxide $(\mathrm{NaOH})$ to obtain a $\mathrm{pH}$ of about $7.4^{9,10}$ (Fig. 2A and 2B).

\section{Freeze Drying and Scanning Electron Microscopy}

The obtained hydrogel was dried under freeze dryer (CHRIST Alpha 1-4 LD plus, Germany) at $-50^{\circ} \mathrm{C}$ and $150 \mu \mathrm{mHg}$ for 24 $\mathrm{h}$ followed by freezing at $70^{\circ} \mathrm{C}$ for $5 \mathrm{~h}$ (Fig. 2C). All samples were kept in room temperature for further analyses. Freeze dried samples were analyzed for morphological assessment and pore size evaluation by Scanning Electron Microscopy (SEM, Zeiss) observations. Samples were frozen in liquid nitrogen, fractured and gold coated before scanning. ${ }^{11}$ (Fig. 2D and $2 \mathrm{E}$ ).

\section{Fibroblast and Keratinocyte Culture onto Hydrogel}

Fibroblasts $\left(5 \times 10^{4}\right.$ cells/gel $)$ were centrifuged and resuspended in $100 \mu$ l DMEM (Gibco, Thermo Fisher Scientific) and mixed with $3 \mathrm{ml}$ of prepared hydrogel. The combination of the cell and fibrinogen solution was placed in a 6 -well cell culture plate. Then, thrombin was added to each well and kept at room temperature for $10 \mathrm{~min}$ followed by $1 \mathrm{~h}$ at $37^{\circ} \mathrm{C}$ in an incubator containing $5 \% \mathrm{CO}_{2} \cdot{ }^{10}$ In same procedure, keratinocytes $\left(5 \times 10^{4}\right.$ cells/gel) was prepared separately and added to the top of fibroblast layer in the same process. KGM Medium (Gibco, Thermo Fisher Scientific) added to the well for 1 day and then processed, and paraffin-embedded for histological analysis (Fig. 3A-3C).

\section{Histological Staining and IHC}

The cell seeded scaffold was fixed using $10 \%$ formalin, processed, and paraffin-embedded. Paraffin sections $(3 \mu \mathrm{m})$ were stained with Hematoxylin and Eosin (Sigma-Aldrich, USA) to assess the histological morphology of the fibrin-collagen hydrogel (Fig. 2D and 2E). Masson's trichrome staining was performed to distinguish the collagen content in the hydrogel
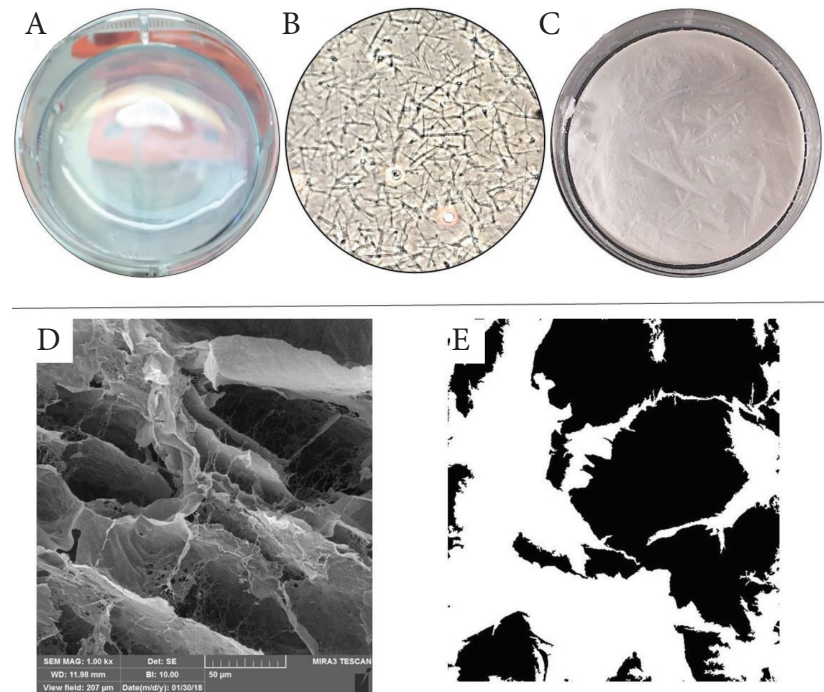

Fig. 2 Scaffold preparation and characterization. (A) Typical view of fibrin-collagen hydrogel. (B) Trilaminar fibrin network after gelation. (C) Freeze dried hydrogel. (D) Representative SEM image of fibrin-collagen hydrogel (scale bars: $50 \mu \mathrm{m}$ ). (E) ImageJ analysis of SEM image to analysis pore size and area.

with cells. ${ }^{12}$ (Fig. 2F). Sections of paraffin-embedded hydrogel which contain keratinocyte and fibroblast cells were deparaffinized (xylol, $5 \mathrm{~min}$ ), dehydrated (ethanol 100-96-80-7050\%, 2 min each), and washed in PBS (5 min). The sections were incubated for $30 \mathrm{~min}$ in $0.3 \%$ Triton X-100 (Sigma-Aldrich, USA), and blocked in 10\% BSA in PBS (Sigma-Aldrich, USA) for $30 \mathrm{~min}$. Immunostaining was performed using a primary antibody, CK14 (BD Bioscience, USA) for keratinocytes, Vimentin (Abcam, USA) for fibroblasts cells seeded into hydrogel followed by a FITC-conjugated secondary antibody (Dako, Switzerland). Pictures of immunofluorescence staining were taken with the FITC-filter sets in the fluorescent microscope (Olympus, Japan) ${ }^{10}$ (Fig. 3G-L).

\section{Results}

Figure 1 shows the morphology of fibroblast cells from the dermis and keratinocytes from the epidermis. Expression of the cell markers was analyzed by flow cytometry for CK14 and Epcam FITC-conjugated antibodies for keratinocyte cells and CD90 and Vimentin for fibroblast cells. As shown, the expression of CK14 and EpCAM differ in different culture media at $75.2 \%$ and $0.341 \%$, respectively, for cells cultured with KGMGold Keratinocyte Growth Medium SingleQuot (Lonza; Sweden). The CK14 and EpCAM markers measured by flow cytometry for keratinocyte cells cultured with Keratinocyte Growth Medium (Epilife Medium; Thermo Fisher Scientific) were $0.087 \%$ and $98.5 \%$, respectively. CD90 and Vimentin for fibroblast cells were $99.2 \%$ and $99.6 \%$, respectively.

We isolated and cultured keratinocyte from the basal layer of the epidermis and cornified layer. As expected, the length of viability were different for fibrin-collagen hydrogels. Cells from the basal layer contain proliferative keratinocytes which have unlimited self-renewal. Unlike keratinocytes from the cornified layer, basal layer keratinocyte cells were able to proliferate for more than one month in a hydrogel scaffold. SEM images of freeze dried hydrogel without cells are shown in Fig. 2D. In this image, high porosity and open interconnected geometry of the 

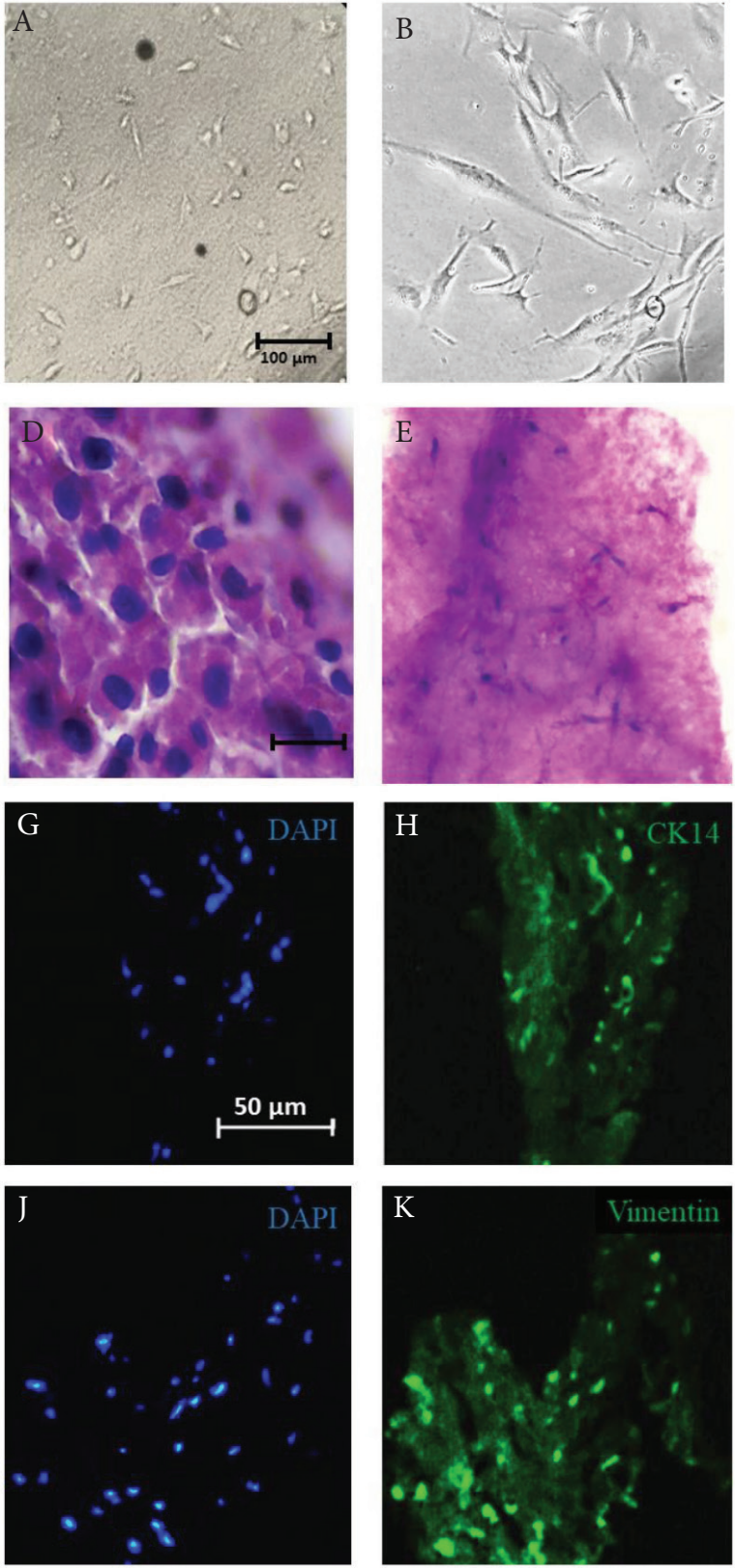
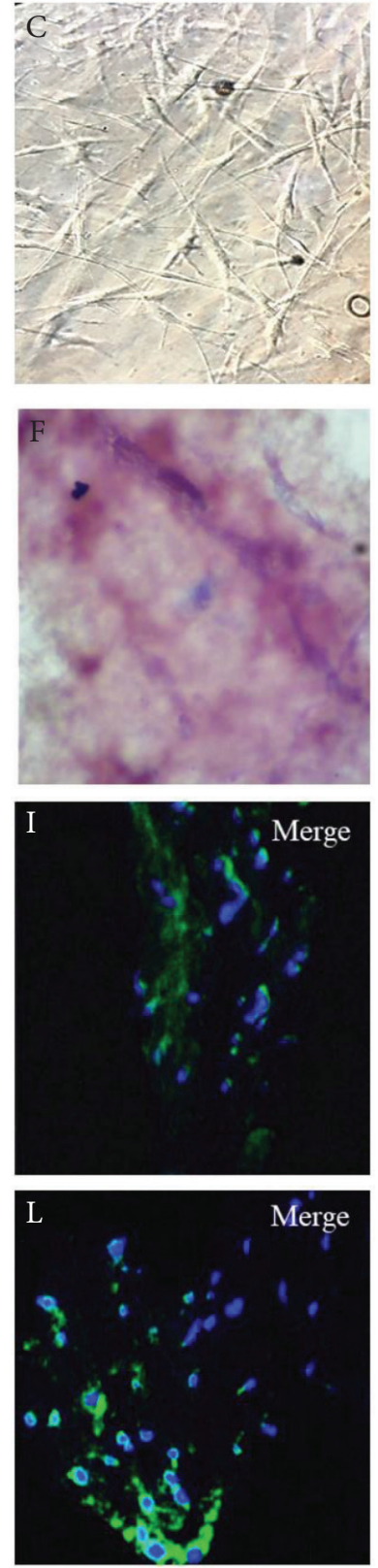

Fig. 3 Three-dimensional skin cell culture. (A-C) Phase-contrast image of fibroblasts at the time of seeding and 7 days after cell seeding into hydrogel (Scale bar: $100 \mu \mathrm{m}$ ). (D and E) Hematoxylin/eosin staining of hydrogel contain keratinocyte cell at the time of seeding and 7 days after cell seeding into hydrogel (Scale bar: $100 \mu \mathrm{m}$ ). (F) Masson's trichrome staining to show collagen content of hydrogel. (G-L) Immunofluorescence staining for human CK14 and vimentin show two layers of skin substitute at day 7 after cell seeding. DAPI stains the nuclei in blue. (Scale bar $=\mathbf{5 0} \mu \mathrm{m})$. pores were observed which allowed cells to become trapped in the scaffold and migrate through it successfully. ImageJ analysis of the SEM image is shown in Fig. 2E, where $51.086 \%$ of the area is composed of pores and the mean $\pm \mathrm{SD}$ of the size of the pore is 42.38 Feret diameter. Despite the advantages of fibrin as a bioscaffold for skin engineering, the application of fibrin-based scaffolds is limited due to their poor mechanical properties and fast biodegradability. ${ }^{13}$

Figs. 3A and 3D shows cells at the time of seeding and at 3 and 7 days after seeding onto hydrogel (Figs. 3B and 3C). These images reveal that cells can proliferate after encapsulation and have suitable migration during $3 \mathrm{D}$ culture. The collagen is added to the fibrin to address this problem. Fibrin-collagen hydrogels were thus prepared as described above and Masson trichrome staining confirmed the presence of collagen in the scaffold (Fig. 3F). Immunofluorescence staining for fibroblasts and keratinocyte cells stained with CK14 and Vimentin (Figs. 3G-3L) was present after cell seeding on day 7 .

\section{Discussion}

Hydrogels were first introduced by Wichterle and $\mathrm{Lim}^{5,14}$ as crosslinked 2-hydroxyethyl methacrylate hydrogels and have been applied in numerous applications because of their hydrophilic features. Subsequently, synthetic and natural hydrogels have been fabricated using different materials. ${ }^{15,16}$ Some features of hydrogel can promote epithelialization of the wound and make hydrogel of interest as a wound dressing. Exudate uptake from bed wounds promotes fibroblast proliferation and keratinocyte migration, efficient delivery (slow release) of bioactive molecules (antimicrobial agents, biomacromolecules, growth factors and other small molecule agents) to the wound bed. ${ }^{7,17}$ Hydrogels are the best choice compared with other dressings because of their elasticity and flexibility, which are requirements of ideal wound dressings.

One of the most promising areas for hydrogels is tissue engineering. Three-dimensional networks of hydrogel allow desirable cellular encapsulation, proliferation and survival, 
biodegradability, biocompatibility and similarities to the natural extracellular matrix that are unique in comparison with other scaffolds. ${ }^{18}$ Despite these advantages and the weak mechanical properties, a major challenge to hydrogel scaffolds is the difficulty associated with engineering complex tissues with multiple cell types, especially as a bioengineered skin substitute. Therefore, hydrogel scaffolds developed for tissue engineering should typically be assessed for their properties. ${ }^{18,19}$

Biological, physicochemical, and topographical surface characteristics of scaffolds are vital parameters in controlling and affecting cellular adhesion and proliferation. ${ }^{20}$ In this study, we present a natural composition of hydrogel for skin cell culture. The engineered hydrogels have been synthesized using self-assembled fibrin-collagen which mimics the components of the native ECM. Fibrin-based biomaterials as cell carriers have been widely investigated in recent decades. ${ }^{21}$ Injectable fibrin gel known as fibrin glue has been investigated more than other forms. ${ }^{22,23}$ Less attention has been paid to fibrin as a scaffold for skin regeneration due to its low mechanical properties. ${ }^{24}$

Because of the important role of fibrin, it can be a suitable scaffold to accelerate wound healing. We investigated a method of natural crosslinking with collagen type I to enhance the mechanical features of fibrin. The trilaminar structure of fibrin after fabrication was visualized by phase-contrast microscopy in Fig. 2B. We show here that the optimal concentration of hydrogel was isolated and cultivated skin cells could be seeded on it as a 3D scaffold. Kretzschmar et al. ${ }^{25}$ and Watt ${ }^{26}$ showed that, within the epidermis, proliferation takes place in the basal layer of keratinocytes having unlimited self-renewal capacity that expresses different cell markers in comparison with other layers. In organotypic cultures, keratinocytes from the cornified layer are cultivated at the airliquid interface. ${ }^{27}$

In this study, keratinocytes were cultivated from different layers of the epidermis. The study shows that basal cell layer keratinocytes are more vital in a 3D cell culture onto hydrogel in comparison with the cornified cell layer. Flow cytometry of select markers revealed differences between keratinocyte cells and high expression of fibroblast cell markers. ${ }^{28}$ Hematoxylin and eosin staining of a hydrogel at the time of seeding and after cell seeding revealed that fibrin-collagen hydrogel is suitable as a scaffold. Importantly, the potential of fibrin-collagen hydrogel for tissue-engineered dermal and epidermal skin grafts after 3D cell culture were confirmed by immunofluorescent staining. These results show improvement in maintaining skin cells in 3D hydrogels as a next generation skin equivalent for the treatment of full-thickness defects.

\section{Conclusion}

Accelerating hydrogel-based dressings have attracted considerable research interest due to their unusual properties and various applications. The combination with hydrogels show potential on full-thickness wounds with irregular margins, although further study is necessary to evaluate different synthetic and natural materials in a clinical setting for enhanced skin regeneration. Further studies should be planned to address a novel method of fabrication that is cost-effective, effective for drug loading, fixing of defects in design or feature, and to improve efficacy. The fibrin-collagen hydrogel offers stability and human fibroblasts and keratinocytes provide viable components to produce dermal and epidermal skin replacement. In an ongoing study, we have recently developed a fibrin-collagen hydrogel as a scaffold for skin cell culture. We anticipate the completion of this study for the release of these novel results. We believe that, as a fibrin-collagen wound dressing, it has great potential clinical applications for wound healing.

\section{Acknowledgments and Funding Sources}

This project is $\mathrm{PhD}$ thesis belonging to the Kashan University of Medical Sciences and Skin and Stem Cell Research Center, Tehran University of Medical Sciences. The work was supported financially by the Iranian Council for the Development of Stem Cell Sciences and Technologies.

\section{Conflict of Interest}

The authors declare no conflict of interest.

\section{References}

1. Sulivan F. Medtech insight. Cytomedix. 2012. Available from: https://www sec.gov/Archives/edgar/data/1091596/000114420412001334/v244875_ ex99-1.htm.

2. Nilforoushzadeh MA, Sisakht MM, Seifalian AM, Amirkhani MA, Banafshe HR, Verdi J, et al. Regenerative medicine applications in wound care. Curr Stem Cell Res Ther. 2017;12:658-674.

3. Alrubaiy L, Al-Rubaiy KK. Skin substitutes: a brief review of types and clinical applications. Oman Med J. 2009;24:4-6

4. Halim AS, Khoo TL, Mohd Yussof SJ. Biologic and synthetic skin substitutes: an overview. Indian J Plast Surg. 2010;43:S23-S28.

5. Kamoun EA, Kenawy ES, Chen X. A review on polymeric hydrogel membranes for wound dressing applications: PVA-based hydrogel dressings. J Adv Res. 2017:8:217-233.

6. Akhtar MF, Hanif M, Ranjha NM. Methods of synthesis of hydrogels ... A review. Saudi Pharm J. 2016;24:554-559.

7. Madaghiele M, Demitri C, Sannino A, Ambrosio L. Polymeric hydrogels for burn wound care: advanced skin wound dressings and regenerative templates. Burns Trauma. 2014;2:153-161.

8. Parenteau NL, Bilbo P, Nolte CJ, Mason VS, Rosenberg M. The organotypic culture of human skin keratinocytes and fibroblasts to achieve form and function. Cytotechnology. 1992;9:163-171.
9. Klar AS, Guven S, Zimoch J, Zapiorkowska NA, Biedermann T, BottcherHaberzeth S, et al. Characterization of vasculogenic potential of human adipose-derived endothelial cells in a three-dimensional vascularized skin substitute. Pediatr Surg Int. 2016;32:17-27.

10. Klar AS, Guven S, Biedermann T, Luginbuhl J, Bottcher-Haberzeth S, Meuli-Simmen C, et al. Tissue-engineered dermo-epidermal skin grafts prevascularized with adipose-derived cells. Biomaterials. 2014;35: 5065-5078.

11. Simoni RC, Lemes GF, Fialho S, Goncalves OH, Gozzo AM, Chiaradia V, et al. Effect of drying method on mechanical, thermal and water absorption properties of enzymatically crosslinked gelatin hydrogels. An Acad Bras Cienc. 2017:89:745-755

12. Cohen AH. Masson's trichrome stain in the evaluation of renal biopsies. Am J Clin Pathol. 1976;65:631-643.

13. Ahmed TA, Dare EV, Hincke M. Fibrin: a versatile scaffold for tissue engineering applications. Tissue Eng Part B Rev. 2008;14:199-215.

14. Gibs I, Janik H. Review: synthetic polymer hydrogels for biomedical applications. Chem Chem Technol. 2010;4:297-304.

15. Bracaglia LG, Messina M, Winston S, Kuo CY, Lerman M, Fisher JP. 3D printed pericardium hydrogels to promote wound healing in vascular applications. Biomacromolecules. 2017;18:3802-3811. 
16. Hu Y, Zhang Z, Li Y, Ding X, Li D, Shen C, et al. Dual-crosslinked amorphous polysaccharide hydrogels based on chitosan/alginate for wound healing applications. Macromol Rapid Commun. 2018;39:e1800069.

17. Annabi N, Rana D, Shirzaei Sani E, Portillo-Lara R, Gifford JL, Fares MM, et al. Engineering a sprayable and elastic hydrogel adhesive with antimicrobial properties for wound healing. Biomaterials. 2017;139:229-243.

18. El-Sherbiny IM, Yacoub MH. Hydrogel scaffolds for tissue engineering Progress and challenges. Glob Cardiol Sci Pract. 2013;2013:316-342.

19. Shin H, Jo S, Mikos AG. Biomimetic materials for tissue engineering. Biomaterials. 2003;24:4353-4364.

20. Boyan BD, Hummert TW, Dean DD, Schwartz Z. Role of material surfaces in regulating bone and cartilage cell response. Biomaterials. 1996;17:137-146.

21. LiY, Meng H, Liu Y, Lee BP. Fibrin gel as an injectable biodegradable scaffold and cell carrier for tissue engineering. ScientificWorld Journal. 2015;2015:685690.

22. Christman KL, Fok HH, Sievers RE, Fang Q, Lee RJ. Fibrin glue alone and skeletal myoblasts in a fibrin scaffold preserve cardiac function after myocardial infarction. Tissue Eng. 2004;10:403-409.
23. Cakmak O, Babakurban ST, Akkuzu HG, Bilgi S, Ovali E, Kongur M, et al. Injectable tissue-engineered cartilage using commercially available fibrin glue. Laryngoscope. 2013;123:2986-2992.

24. Litvinov RI, Weisel JW. Fibrin mechanical properties and their structural origins. Matrix Biol. 2017;60-61:110-123.

25. Kretzschmar K, Watt FM. Markers of epidermal stem cell subpopulations in adult mammalian skin. Cold Spring Harb Perspect Med. 2014;4. pii: a013631.

26. Watt FM. Epidermal stem cells: markers, patterning and the control of stem cell fate. Philos Trans R Soc Lond B Biol Sci. 1998;353:831-837.

27. Schoop VM, Mirancea N, Fusenig NE. Epidermal organization and differentiation of HaCaT keratinocytes in organotypic coculture with human dermal fibroblasts. J Invest Dermatol. 1999;112:343-353.

28. Pontiggia L, Biedermann T, Meuli M, Widmer D, Bottcher-Haberzeth S, Schiestl C, et al. Markers to evaluate the quality and self-renewing potential of engineered human skin substitutes in vitro and after transplantation. J Invest Dermatol. 2009;129:480-490.

This work is licensed under a Creative Commons Attribution-NonCommercial 3.0 Unported License which allows users to read, copy, distribute and make derivative works for non-commercial purposes from the material, as long as the author of the original work is cited properly. 